\title{
Probability Density
}

National Cancer Institute

\section{Source}

National Cancer Institute. Probability Density. NCI Thesaurus. Code C94958.

A quantifier used to characterize a statistical distribution. It describes the probability of a

variable having a value within a small unit band of values around this value. 